\title{
Effects of Feedback-Based Visual Line- Orientation Discrimination Training for Visuospatial Disorders After Stroke
}

Neurorehabilitation and

Neural Repair

27(2) $142-152$

(C) The Author(s) 2013

Reprints and permission: http://www. sagepub.com/journalsPermissions.nav DOI: 10.1 I 77/I5459683।2457826

http://nnr.sagepub.com

\begin{abstract}
Background. Patients with right or more rarely left parietotemporal lesions after stroke may have profound visuospatial disorders that impair activities of daily living (ADL) and long-term outcome. Clinical studies indicate improvements with systematic training of perception. Studies of perceptual learning in healthy persons suggest rapid improvements in perceptual learning of spatial line orientation with partial transfer to nontrained line orientations. Objective. The authors investigated a novel feedback-based perceptual training procedure for the rehabilitation of patients after stroke. Methods. In an uncontrolled trial, 13 participants showing profound deficits in line orientation and related visuospatial tasks within 12 to 28 weeks of onset performed repetitive feedback-based, computerized training of visual line orientation over4 weeks of treatment. Visual line-orientation discrimination and visuospatial and visuoconstructive tasks were assessed before and after training. Results. The authors found (a) rapid improvements in trained but also in nontrained spatial orientation tests in all 13 participants, partially up to a normal level; (b) stability of the obtained improvements at 2-month follow-up; (c) interocular transfer of training effects to the nontrained eye in 2 participants suggesting a central, postchiasmatic locus for this perceptual improvement; and $(d)$ graded transfer of improvements to related spatial tasks, such as horizontal writing, analog clock reading, and visuoconstructive capacities but no transfer to unrelated measures of visual performance. Conclusions. These results suggest the potential for treatment-induced improvements in visuospatial deficits by feedbackbased, perceptual orientation training as a component of rehabilitation after stroke.
\end{abstract}

\section{Keywords}

visuospatial disorders, hemispatial neglect, recovery of function, stroke rehabilitation

\section{Introduction}

Focal brain damage after stroke can be accompanied by profound visuospatial disorders. ${ }^{1-3}$ Lesions of extrastriate cortical and subcortical structures-for example, parietal, temporoparietal, thalamic, or basal ganglia lesions of the right $(50 \%-70 \%)$ or left hemisphere $(30 \%-50 \%)$-lead to impairments in space perception. ${ }^{4}$ Typically, righthemispheric lesions cause more frequent and more severe deficits compared with left-hemispheric lesions. ${ }^{5,6}$ Deficits following such lesions include impairments in lineorientation discrimination ${ }^{7,8}$; the subjective vertical ${ }^{9}$; line bisection and subjective straight ahead ${ }^{10}$; size, distance, and position estimation ${ }^{11}$; clock reading/drawing ${ }^{12}$; and block-design performance. ${ }^{13}$

Deficits in visual orientation discrimination most frequently occur after lesions affecting the parietal cortex and/ or the basal ganglia. ${ }^{6,7}$ Apart from the parietal cortex, ${ }^{14-17}$ there is evidence for a distributed network underlying orientation processing. ${ }^{18}$

\section{Rehabilitation of Visuospatial Disorders}

Visuospatial disorders often lead to impairments in activities of daily living (ADL) and show adverse effects on therapy outcome. $^{4,19}$ Many patients with large right-hemispheric

\footnotetext{
'Ludwig Maximilian University, Munich, Germany

${ }^{2}$ Clinical Neuropsychology Unit, Saarland University, Saarbruecken, Germany

${ }^{3}$ International Research Training Group 1457 "Adaptive Minds," Saarbruecken, Germany

${ }^{4}$ Erlangen University, Erlangen, Germany

${ }^{5}$ Mediclin Neurological Rehabilitation Clinic Bosenberg, St Wendel, Germany
}

\section{Corresponding Authors:}

Johanna Funk, PhD, General and Experimental Psychology, Ludwig Maximilian University, Leopoldstr I3, D-80802 Munich, Germany Email:Johanna.Funk@psy.Imu.de

Georg Kerkhoff, PhD, Clinical Neuropsychology Unit, Saarland University, Im Stadtwald Building A I3, D-66I23 Saarbruecken, Germany E-Mail: kerkhoff@mx.uni-saarland.de 
lesions show combinations of visuospatial deficits, spatial neglect, and anosognosia, ${ }^{20,21}$ the latter being an especially difficult therapeutic problem. ${ }^{22}$ Neurorehabilitation research has primarily dealt with spatial neglect, ${ }^{23}$ showing effects of neck muscle vibration, ${ }^{24}$ prism adaptation, ${ }^{25,26}$ and vestibular, ${ }^{27}$ optokinetic, ${ }^{28,29}$ and theta burst stimulation. ${ }^{30}$ Yet there is also evidence suggesting some efficacy of visuospatial and visuoconstructive therapy on visuospatial impairments apart from neglect. Successful therapeutic approaches for visuospatial disorders include spatial-perceptual training by feedback, ${ }^{20,31}$ visuoconstructive training, ${ }^{13}$ and ADL therapy. For instance, visuospatial deficits were found to be reduced via the graded training of spatial-perceptual capacities with verbal feedback, which is assumed to recalibrate spatial perception. $^{31}$ Still, only few treatments for visuospatial deficits apart from neglect have been evaluated quantitatively.

\section{Perceptual Learning and Plasticity}

Perceptual learning involves relatively long-lasting changes to an organism's perceptual system that improve its ability to respond to its environment. ${ }^{32}$ In unaffected controls, effects of perceptual learning on various perceptual and cognitive functions have been documented. ${ }^{32,33}$ Visual perception was shown to improve with practice in several tasks, including orientation discrimination. ${ }^{34-36}$ However, the underlying neural mechanisms are not fully understood, and the literature is inconsistent regarding restrictions of learning. Some orientation learning studies found $n^{34}$ or only limited ${ }^{36}$ transfer to unpracticed orientations or other retinal locations, ${ }^{34}$ suggesting that perceptual learning occurs at early stages of processing and is restricted to sensory areas addressed by an individual stimulus/task. ${ }^{37-39}$ Other studies found that improvements can generalize, ${ }^{37,40}$ depending on the trained function and task complexity. ${ }^{41}$ For instance, contrast sensitivity training in patients with amblyopia was shown to transfer to other visual functions, ${ }^{40}$ indicating plasticity at higher levels of visual processing. The generalization of training effects is important for understanding the neural mechanisms underlying learning but also serves as an indicator of the practical value of training procedures. Findings of transfer effects encourage the use of perceptual learning in neurorehabilitation. Thereby, it is necessary to identify factors critical for training success and transfer of training to further functions and tasks.

In unaffected controls, trialwise feedback was shown to be a potential factor of training success. ${ }^{42}$ Feedback enhanced training effects for oblique but not cardinal directions, indicating that feedback might be important to sharpen the representation of oblique stimuli but less important for training cardinal directions, which already have a more stable representation. These results indicate that feedback enhances training benefits especially when representations of stimuli are unstable, as they are in participants with visuospatial disorders related to orientation representation. Especially when disorders are accompanied by anosognosia, feedback might be important in rehabilitation to improve the insight into quality and magnitude of visuospatial deficits.

This study investigated the effects of repetitive feedback-based line-orientation training on orientation discrimination in patients with severe visuospatial deficits after recent stroke. The main objective was (1) to investigate the effectiveness of such a perceptual training to improve visual orientation discrimination in stroke patients and to assess whether potential training effects generalize to all orientations and persist over time. (2) In order to shed light on the putative stage(s) of processing affected by the training, interocular transfer was measured in two subjects. (3) Furthermore, transfer effects to related visuospatial and visuoconstructive parameters relevant to ADL performance as well as to visual tasks unrelated to orientation discrimination were measured to assess generalization and specificity of the treatment.

\section{Methods}

\section{Participants}

A total of 13 participants with single, vascular lesions and no evidence of brain stem lesions (revealed by CT/MRI) were included in the study - 11 with right-hemispheric and 2 with left-hemispheric lesions. Participants were recruited within a neuropsychological outpatient department based on the following criteria: $(a)$ single, unilateral supratentorial stroke and $(b)$ deficits in at least 2 of 4 tests described below: Benton Judgment of Line Orientation Test (JLOT), ${ }^{43}$ $<22$ nd percentile; Mack-Levine Test, ${ }^{44}<7$ correct items; analog clock reading, $>2$ errors; $45^{\circ}$ orientation discrimination, $>8^{\circ}$ uncertainty interval. Exclusion criteria were (a) bilateral lesions, (b) severe aphasia precluding proper comprehension of instructions, and (c) psychiatric problems. Binocular visual fields were mapped with kinetic perimetry. ${ }^{45}$ Neglect severity was measured in a screening (on white paper $29.7 \times 19.7 \mathrm{~cm}^{2}$ ) that included copy drawing (star, daisy, clock, house, and face), horizontal line bisection $(20 \mathrm{~cm} \times 1 \mathrm{~mm}$ black line $)$, number cancellation (10 targets in each hemispace among 100 numbers on the sheet), and a reading test with 180 words sensitive to neglect. ${ }^{46}$ The coexistence of neglect was no criterion for selection. Table 1 summarizes the demographic and clinical data. In all, 10 participants had parietal cortical lesions, and 3 had thalamus or basal ganglia lesions. The mean age was 45.6 years (23-60 years), "mean time since lesion onset was 20.7 weeks (12-28 weeks). 12 participants were right handed and 1 left handed. All had normal or corrected-to-normal visual acuity. No participant had disease of the anterior visual pathways as judged from orthoptic/ophthalmological investigations (fundus examination, slit lamp); 9 participants had left-sided homonymous visual field deficits ( 7 hemianopia, 
Table I. Demographic and Clinical Data of Brain-Damaged Participants

\begin{tabular}{|c|c|c|c|c|c|c|c|c|c|c|c|}
\hline Patien & tAge, y & Sex & Educ, $y$ & Etiology & Lesion Locus & TSO,wk & Hemiplegia & $\begin{array}{l}\text { Visual Field/Field } \\
\text { Sparing, deg }\end{array}$ & $\begin{array}{c}\text { Visual } \\
\text { Neglect }^{\mathrm{a}}\end{array}$ & $\begin{array}{c}\text { Training } \\
\text { Sessions } \\
\text { (Trials) }\end{array}$ & $\begin{array}{l}\text { Training } \\
\text { Type }\end{array}$ \\
\hline 1 & 40 & $\mathrm{~F}$ & 10 & $\begin{array}{l}\mathrm{ICH} \text { right, } \\
\mathrm{MCl} \text { right }\end{array}$ & Parietal & 12 & L & Left, hemianopia, $I^{\circ}$ &,$+++ L$ & $14(22 \mid)$ & Binocular \\
\hline 2 & 32 & $\mathrm{~F}$ & 8 & $\mathrm{MCl}$ right & Parietal & 12 & - & Normal &,$++ \mathrm{L}$ & II (224) & Binocular \\
\hline 3 & 42 & $\mathrm{~F}$ & II & $\mathrm{ICH}$ left, $\mathrm{MCl}$ left & Parietal & 24 & - & Normal &,$+ \mathrm{R}$ & II (245) & Binocular \\
\hline 4 & 41 & $M$ & 13 & $\mathrm{MCl}$ right & Parietal & 24 & $\mathrm{~L}$ & Left, hemianopia, $2^{\circ}$ &,$+++ L$ & $19(504)$ & Monocular \\
\hline 5 & 60 & $M$ & 8 & $\mathrm{MCl}$ right & Parietal & 24 & L & Normal &,$+++ L$ & $17(525)$ & Binocular \\
\hline 6 & 45 & $F$ & 12 & $\mathrm{ICH}$ right & Frontoparietal & 24 & L & $\begin{array}{l}\text { Left, impaired form/ } \\
\text { color perception, } 20^{\circ}\end{array}$ &,$++ \mathrm{L}$ & $13(250)$ & Binocular \\
\hline 7 & 57 & M & 11 & $\begin{array}{l}\mathrm{PCl} \text { (calcarina) } \\
\text { right, ICH right }\end{array}$ & $\begin{array}{l}\text { Occipital, } \\
\text { thalamic }\end{array}$ & 24 & L & Left, hemianopia, $2^{\circ}$ &,$+ \mathrm{L}$ & $10(188)$ & Binocular \\
\hline 8 & 54 & $M$ & 14 & $\mathrm{ICH}$ right & Parietal & 12 & - & Left, hemianopia, $I^{\circ}$ &,$++ \mathrm{L}$ & $10(276)$ & Binocular \\
\hline 9 & 59 & $M$ & 17 & $\begin{array}{l}\mathrm{ICH} \text { right } \\
\text { (putamen, } \\
\text { claustrum) }\end{array}$ & Basal ganglia & 24 & $\mathrm{~L}$ & Left, hemianopia, $2^{\circ}$ &,$+++ L$ & II (247) & Monocular \\
\hline 10 & 57 & $M$ & 10 & $\mathrm{MCl}$ right & $\begin{array}{c}\text { Temporo- } \\
\text { occipitoparietal }\end{array}$ & 24 & - & $\begin{array}{l}\text { Left, impaired form/ } \\
\text { color perception, } 24^{\circ}\end{array}$ &,$+ \mathrm{L}$ & $6(107)$ & Binocular \\
\hline II & 52 & $M$ & 9 & $\mathrm{MCl}$ right & Frontoparietal & 17 & - & Left, hemianopia, $I^{\circ}$ &,$++ \mathrm{L}$ & $7(143)$ & Binocular \\
\hline 12 & 23 & $\mathrm{~F}$ & 12 & $\begin{array}{l}\mathrm{ICH} \text { right } \\
\text { thalamus }\end{array}$ & Thalamic & 28 & L & Left, hemianopia, $7^{\circ}$ &,$++ \mathrm{L}$ & $10(135)$ & Binocular \\
\hline 13 & 31 & $\mathrm{~F}$ & 8 & $\mathrm{MCl}$ left & Inferior parietal & 20 & - & Normal &,$+ \mathrm{R}$ & $8(170)$ & Binocular \\
\hline
\end{tabular}

Abbreviations: F, female; M, male; Educ, education (years of schooling); $\mathrm{MCl} / \mathrm{PCl}$, middle/posterior cerebral artery infarction; ICH, intracerebral hemorrhage; TSO, time since onset; L/R, left/right.

${ }^{a}$ Visual neglect severity: + , signs of mild contralateral visual neglect in at least 2 of 4 screening tests (copy drawing, line bisection, number cancellation, and text reading); ++ , moderate contralateral visual neglect in 3 of 4 neglect screening tests; +++ , severe contralateral visual neglect in all 4 neglect screening tests.

2 impaired form/color perception in the contralesional visual field). Aphasia was ruled out in participants with left-hemispheric damage on the basis of the Aachen Aphasia test. ${ }^{47}$ All participants showed profound visuospatial disorders and mild to severe visual neglect. Informed consent according to the Declaration of Helsinki II was obtained from all participants. The study was approved by the local ethics committee (Bavarian Medical Association).

\section{Visual Line-Orientation Discrimination}

Visuospatial measurements were taken in darkness with the chassis of the PC monitor covered by an oval-shaped mask to eliminate visual reference cues. Participants were tested at a $0.5-\mathrm{m}$ viewing distance with spectacle corrections where necessary. Head position was stabilized by a headand-chin rest. Visual orientation discrimination was measured with the computerized visual-spatial perception program (VS) ${ }^{48}$ Participants viewed 2 oblique lines $(10 \mathrm{~cm}$ $\times 1.4 \mathrm{~mm}$ ) oriented differently on the screen. The experimenter rotated 1 line via mouse clicks until participants indicated that both lines had the same orientation. Based on the methods of limits, ${ }^{49}$ the psychophysical parameters constant error (difference between target and reference line at the point of subjective equality) and interval of uncertainty (range in which lines are perceived as being parallel) were calculated by the program. Visual orientation discrimination was measured for 6 angles: $30^{\circ}, 45^{\circ}$, and $60^{\circ}$ (clockwise) and $120^{\circ}, 135^{\circ}$, and $150^{\circ}$ (counterclockwise). Furthermore, the subjective visual vertical and horizontal (SVV, SVH) were measured via VS. Retest reliability for the $45^{\circ}$ orientation discrimination in VS is 0.86 , and retest reliability of the SVV is $0.84 .^{50}$ The step width was $0.5^{\circ}$ in all measurements. There were 10 trials for each orientation. Orientation conditions were blocked, and the sequence of blocks was counterbalanced to control for practice effects. The starting position of the target line was $20^{\circ}$ from the orientation of the reference line. The direction of initial tilt was counterbalanced to control for effects of rotation direction. Before testing, participants performed 5 practice trials.

\section{Testing Sequence and Training Procedure}

Participants performed the described procedure in 2 baseline sessions before training and in 1 posttraining session. The second baseline was collected 6 weeks after the first baseline to control for effects of spontaneous remission. After the second baseline, participants completed 4 weeks 


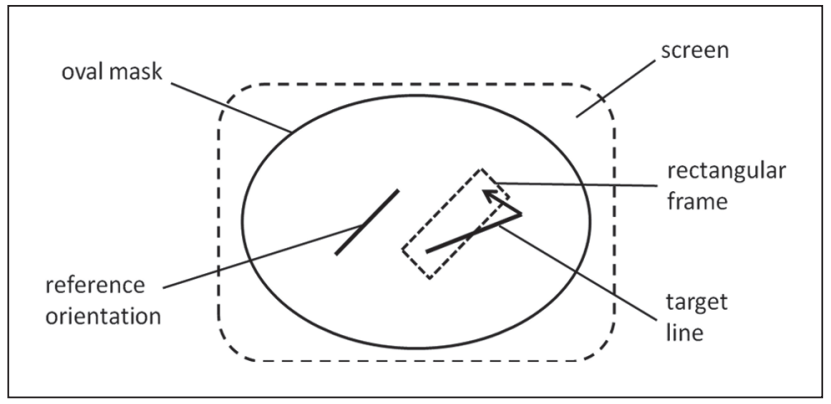

Figure I. Experimental setup in the visual line-orientation discrimination training.

of training, followed by the posttraining session. Additionally, participants performed a follow-up session (only for the $45^{\circ}$ and $135^{\circ}$ orientation) 8 weeks after the posttraining session. In the 4 weeks of training, participants practiced the discrimination of the $45^{\circ}$ orientation and received perceptual feedback to train the correct orientation (Figure 1). Visual feedback was given via a rectangular frame around the target line, which became progressively narrow in the course of training (initial size $=20^{\circ}$, final size $=8^{\circ}$, step width $=1^{\circ}$ ). This rectangular frame was presented simultaneously with the line during training. It was green in case of orientation adjustments within the respective range and red when the target line was rotated out of the range. Participants performed 11.3 sessions (249 trials) on average, ranging from 6 to 19 sessions (107-525 trials). Treatment was terminated when $(a)$ normal cutoff scores were reached (orientation discrimination $45^{\circ}$, uncertainty interval $<8^{\circ}$, which was the case in 12 participants) or $(b)$ when no further improvement was observed over 5 consecutive sessions.

\section{Monocular Training}

Two participants with equivalent time since onset (participants 4 and 9) performed a monocular version of the orientation training. In the monocular training, 1 eye was covered by an eye patch in all training sessions. Participant 4 performed 19 training sessions (504 trials) with the left eye only, and participant 9 performed 11 training sessions (247 trials) with the right eye only. The baseline sessions, the posttraining session, and the follow-up session were performed monocularly with the trained and the nontrained eye in both participants to test whether there was transfer of training from 1 eye to the other. In these sessions, participants performed 6 trials for each orientation $\left(30^{\circ}, 45^{\circ}, 60^{\circ}\right.$, $\left.120^{\circ}, 135^{\circ}, 150^{\circ}\right)-3$ with clockwise and 3 with counterclockwise rotation.

\section{Visuospatial and Visuoconstructive Tests Related to Orientation Perception}

To investigate potential transfer of improvements to visuospatial tasks assumed to rely on the integrity of orientation perception, further measures of visuospatial, visuoconstructive, and ADL capacities were obtained in the baseline sessions and the posttraining session.

The JLOT is a visuospatial test ${ }^{43}$ requiring participants to identify the orientation of 2 target lines on a multiplechoice display with 11 reference lines (the difference of each 2 being $18^{\circ}$ ). The test has 2 parallel forms, containing 5 practice and 30 test items, which were alternated between sessions. The number of errors and performance time were measured. Split-half reliability of the JLOT is 0.91 , and retest-reliability is $0.90 .^{51}$

The Mack-Levine test is a visuoconstructive test consisting of 8 items, each containing several pieces that have to be arranged into a square. ${ }^{44}$ Participants had 5 minutes for each item. Task difficulty was manipulated via the number of pieces and the angle/length of their edges. The number of correct items and performance time were scored. This test is highly sensitive for visuoconstructive disorders (18/19 right-hemisphere damaged patients) and correlates significantly with visual orientation discrimination $(r=0.56$; $P<.05)^{44}$

Analog clock reading was assessed in a clock-reading test with 2 practice and 20 test items. Each item displayed a target clock face ( $4 \mathrm{~cm}$ in diameter) on a $29.7 \times 19.7 \mathrm{~cm}^{2}$ sheet of white paper with 4 differently shaped clock faces (oval, octagon, square, and circle). The hands of the target clock face showed a specific time corresponding to 1 of the 4 multiple-choice clock faces, which had to be indicated by the participants. The position of the correct clock face was pseudorandomly alternated. The number of correct items was scored. Performance in this test is significantly correlated with orientation discrimination in the JLOT ( $r=0.78$; $P<.001$; Kerkhoff, unpublished observations).

Horizontal writing was measured to assess spatial dysgraphia. Participants were required to write their names and addresses horizontally, beginning from the very left of a 29.7 $\times 19.7 \mathrm{~cm}^{2}$ sheet of paper. This procedure was repeated 5 times on different sheets. Three participants were unable to perform this task. Deviations from the objective horizontal (in degrees) were measured, and the range of uncertainty and the median of the deviation were calculated.

\section{Visual Tests Unrelated to Orientation Perception}

Further measures of visual performance unrelated to orientation perception were determined before and after the training to test whether potential transfer effects are rather unspecific (eg, enhanced motivation) or limited to tasks related to orientation perception.

Spatial contrast sensitivity was measured via the Cambridge Low Contrast Gratings. ${ }^{52}$ The gratings assess contrast sensitivity at a spatial frequency of $4 \mathrm{c} /$ degree. A series of gratings were shown in order of descending contrast until an error was made. Then, 4 descending series were shown separately to each eye, and a mean sensitivity score was calculated, averaged across both eyes. 


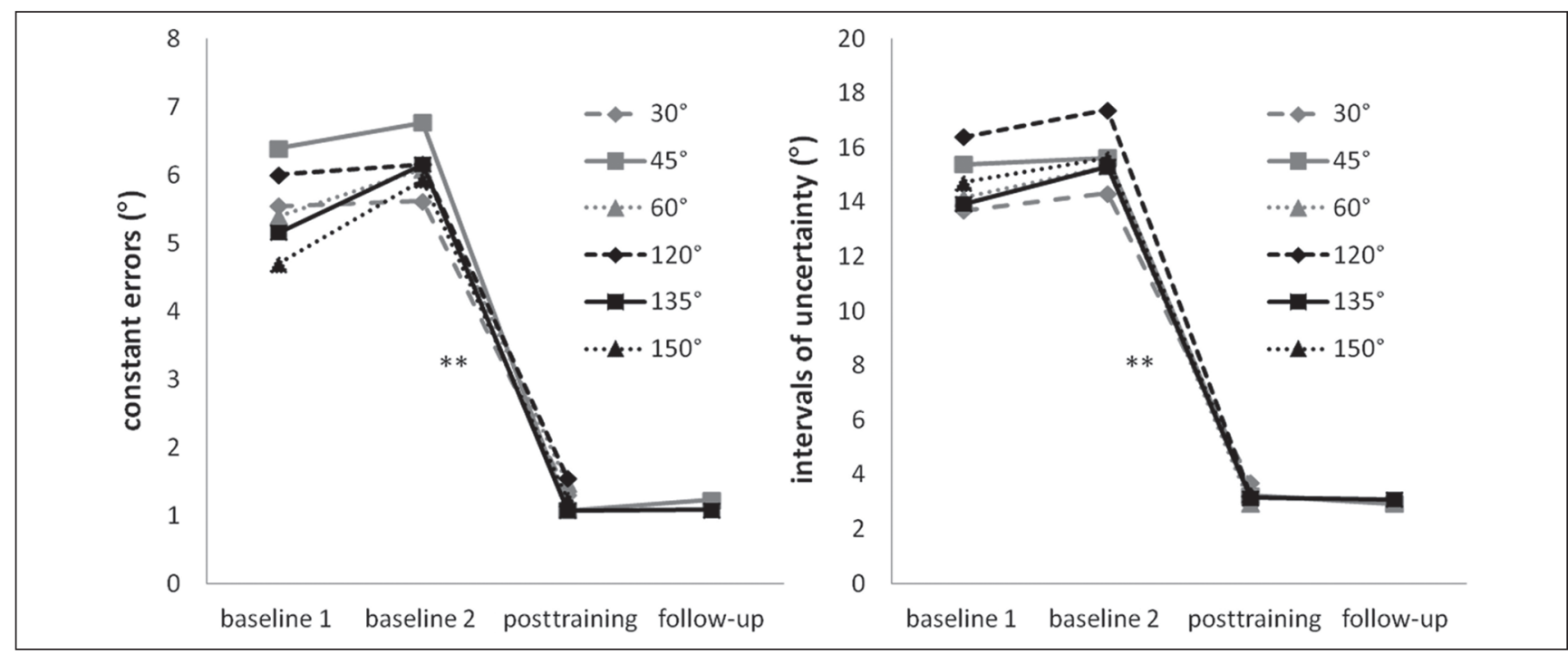

Figure 2. Average visual line-orientation discrimination performance (constant errors and intervals of uncertainty) in the 2 baseline sessions and the posttraining session and follow-up performance for the $45^{\circ}$ and $135^{\circ}$ orientation; positive constant errors indicate counterclockwise tilts of orientation judgments; each line displays I of 6 orientation conditions $\left(30^{\circ}, 45^{\circ}, 60^{\circ}, 120^{\circ}, 135^{\circ}\right.$, and $\left.150^{\circ}\right)$ **Indicates a statistically highly significant difference between baseline sessions and posttraining/follow-up session.

Text reading performance was determined in a 180 word reading test sensitive to neglect and hemianopic reading disturbances. ${ }^{46}$ Reading time was measured in seconds. Alternative 180-word texts were used before and after training.

Visual search performance was measured in a search task on large visual displays. ${ }^{53}$ Search slides were projected on the wall, and the patient had to point at all symbols of a specific class (circles or triangles). The ratio of targets to distractors was $50 \%$. Search time (in seconds) and number of errors (omissions or double detections) were measured.

\section{Statistical Analysis}

Effects of training on orientation discrimination were assessed using repeated-measures analyses of variance (ANOVAs) with the 3-steps factor Training (baseline 1, baseline 2, posttraining) for the constant errors and intervals of uncertainty for all orientations $\left(30^{\circ}, 45^{\circ}, 60^{\circ}, 120^{\circ}\right.$, $135^{\circ}, 150^{\circ}$ ) and the SVV/SVH. To assess the persistence of training effects, constant errors and intervals of uncertainty in a follow-up session (of the $45^{\circ}$ and $135^{\circ}$ orientation only) were compared with performance in the baseline sessions and the posttraining session via paired $t$ tests. Transfer of effects to related visuospatial functions was assessed in equivalent ANOVAs for performance parameters in the $\mathrm{JLOT}^{43}{ }^{4}$ the Mack-Levine test, ${ }^{44}$ analog clock reading, and horizontal writing. To analyze whether transfer effects extend to visual functions unrelated to orientation perception, repeated-measures ANOVAs with the 2-steps factor Training (before and after training) were computed for performance in the contrast sensitivity, text reading, and visual search task. In case of significant main effects or interactions, contrasts were used to compare performance between different sessions. To assess the extent of interocular transfer (in participants 4 and 9), Pearson correlation coefficients were determined, comparing orientation discrimination in the trained and the nontrained eye across training sessions. The $\alpha$ level was chosen as $P<$ .05 for all analyses, corrected for multiple comparisons via Bonferroni corrections.

\section{Results}

\section{Training Effects on Visual Orientation Discrimination}

Significant effects of training on constant errors $(d f=2$, all $F>17.63$, all $P<.001)$ and intervals of uncertainty $(d f=$ 2, all $F>36.31$, all $P<.001$ ) were found for all orientations $\left(30^{\circ}, 45^{\circ}, 60^{\circ}, 120^{\circ}, 135^{\circ}\right.$, and $\left.150^{\circ}\right)$. Both parameters were significantly larger in the baseline sessions compared with the posttraining session (all $P<.001$; Figure 2), whereas performance in the baseline sessions did not differ significantly in all orientation conditions (all $P>.05$, except for the constant errors in the $135^{\circ}$ condition, where the difference between baselines reached $P<.05)$.

\section{Persistence of Training Effects}

In the follow-up session of the $45^{\circ}$ and the $135^{\circ}$ orientation condition, constant errors and intervals of uncertainty were 


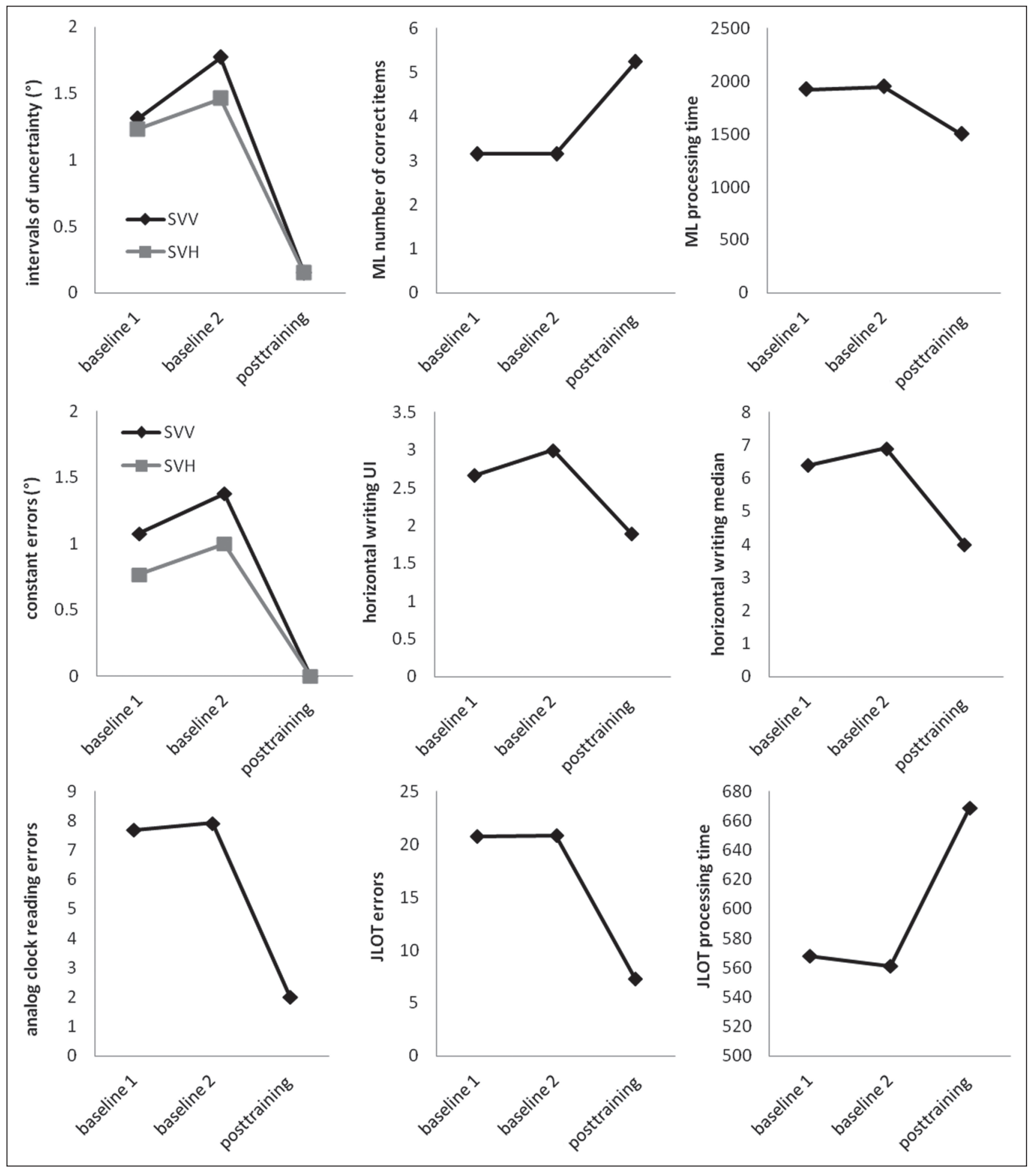

Figure 3. Average performance of patients in the SVV and SVH and measures of visuospatial and visuoconstructive capacities (JLOT, horizontal writing, analog clock reading, Mack-Levine test) for the 2 baseline sessions and the posttraining session. Abbreviations: ML, MackLevine test; UI, uncertainty intervals in horizontal writing; JLOT, Judgment of Line Orientation Test.

significantly smaller than in the baseline sessions (all $P<$ .01 ; Figure 2); in contrast, they did not differ significantly from the posttraining session (all $P>.40$ ), indicating persistent training effects.

\section{Training Effects on SVV and SVH}

Significant effects of training were found on constant errors $(\mathrm{SVV}: d f=2, F=10.15, P<.01 ; \mathrm{SVH}: d f=2, F=8.38 .0$, 

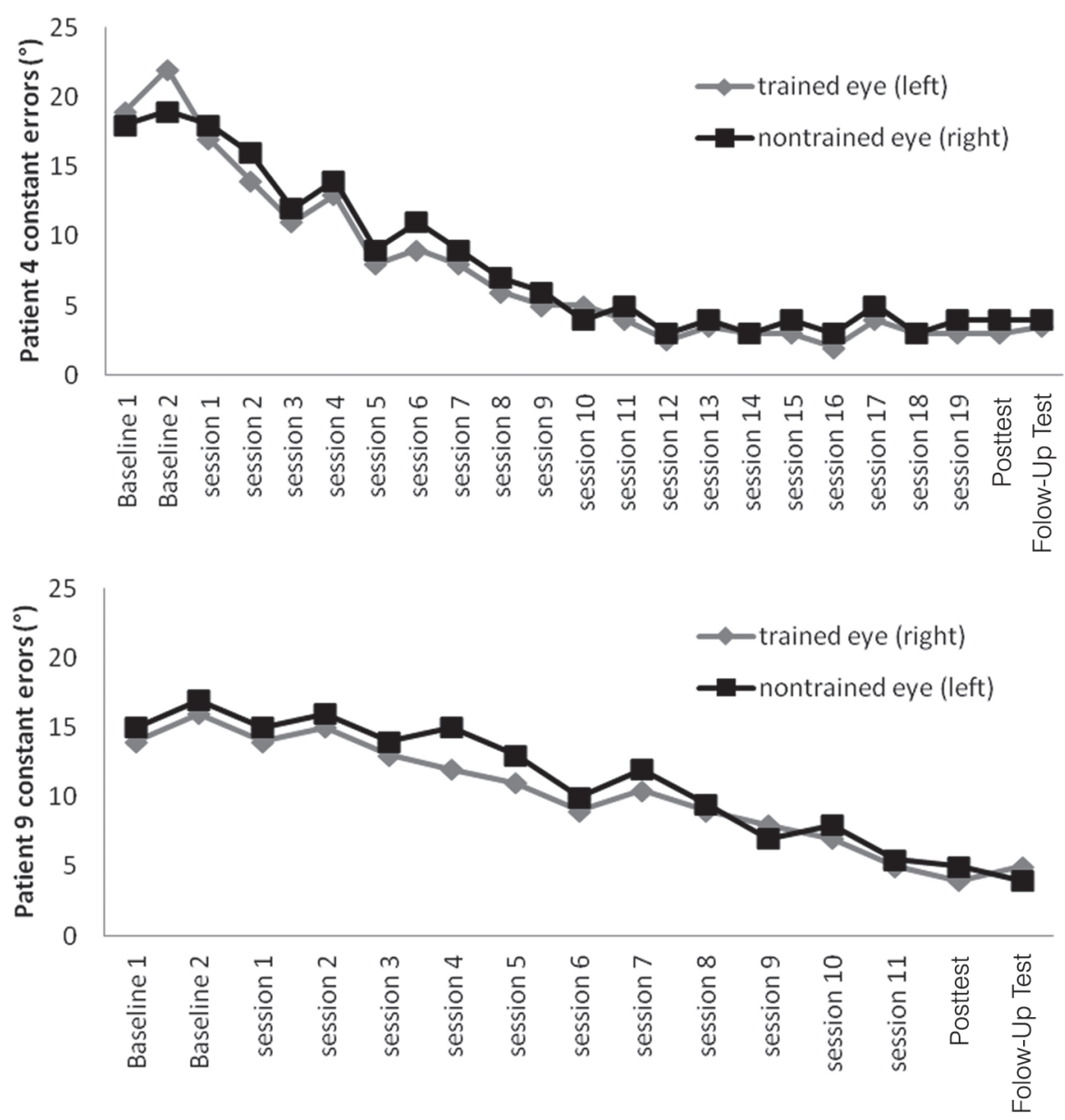

Figure 4. Interocular transfer: average constant errors of patients 4 and 9 across the course of training sessions for the trained and nontrained eyes. One data point represents the average constant error across 6 measurements and 6 orientation conditions (ie, 36 measurements).

$P<.01)$ and intervals of uncertainty (SVV: $d f=2, F=$ $11.17, P<.01$; SVH: $d f=2, F=8.44, P<.01)$. Significantly larger parameters were observed in the baseline sessions compared with the posttraining session (all $P<.01$ ), whereas performance in the baseline sessions did not differ significantly (all $P>.05$; Figure 3 ).

\section{Interocular Transfer}

Figure 4 displays the mean constant errors of participants 4 and 9 across the course of training sessions (11 and 19 sessions, respectively). As can be seen, the curves for the trained and the nontrained eyes are nearly identical. Pearson correlations relating performance of the trained to that of the nontrained eyes across training sessions were highly significant (both $r=0.98 ; P<.01$ ), indicating nearly perfect interocular transfer.

\section{Visuospatial Tests Related to Orientation Perception}

Figure 3 displays the average performance in the visuospatial and visuoconstructive tasks for the 2 baseline sessions and the posttraining session.

For the JLOT, ${ }^{43}$ significant effects of training on the number of errors $(d f=2 ; F=87.57 ; P<.01)$ and percentile ranks $(d f=2 ; F=67.97 ; P<.01)$ and a by-trend significant effect on processing times $(d f=2 ; F=4.28 ; P<.10)$ were revealed. Participants made significantly fewer errors $(P<.01)$ and had higher percentile ranks $(P<.01)$ but had by-trend increased processing times in the posttraining session compared with the baseline sessions $(P<$ $.10)$, whereas performance in the baseline sessions did not differ significantly (all $P>.30$ ).

In the Mack-Levine test ${ }^{44}$ significant effects on the number of correct items $(d f=2 ; F=22.49 ; P<.01)$ and overall 
Table 2. Measures of Visuospatial and Visuoconstructive Capacities Related to Orientation Perception and Visual Capacities Unrelated to Orientation Perception Before/After Training ${ }^{a}$

\begin{tabular}{|c|c|c|c|c|c|c|c|c|}
\hline Patient & $\begin{array}{l}\text { Contrast } \\
\text { Sensitivity }\end{array}$ & $\begin{array}{l}\text { Reading } \\
\text { Time, s }\end{array}$ & $\begin{array}{l}\text { Visual Search } \\
\text { Time, s }\end{array}$ & $\begin{array}{l}\text { Visual Search, } \\
\text { errors }\end{array}$ & $\begin{array}{c}\text { Line-Orientation } \\
\text { Judgment }\end{array}$ & $\begin{array}{c}\text { Visuoconstructive } \\
\text { Disorder }\end{array}$ & $\begin{array}{l}\text { Analog Clock } \\
\text { Reading }\end{array}$ & $\begin{array}{c}\text { Horizonta } \\
\text { Writing }\end{array}$ \\
\hline I & $210 / 150$ & $131 / 139$ & $40 / 37$ & $10 / 9$ & $0 / 22$ & $0 / 3$ & $11 / 1$ & $14 / 9$ \\
\hline 2 & $560 / 560$ & $128 / 138$ & $65 / 67$ & $\mathrm{I} / \mathrm{I}$ & $22 / 57$ & $8 / 8$ & $6 / 1$ & - \\
\hline 3 & $560 / 480$ & $104 / 102$ & $75 / 81$ & $4 / 2$ & $0 / 57$ & $4 / 5$ & $7 / 3$ & - \\
\hline 4 & $480 / 480$ & $|46 /| 40$ & $40 / 41$ & $3 / 3$ & $0 / 11$ & $3 / 3$ & $13 / 3$ & $6 / 6$ \\
\hline 5 & $560 / 560$ & $|33 /| 3 \mid$ & $50 / 42$ & $2 / 2$ & $0 / 57$ & $0 / 5$ & $10 / 1$ & $6 / 4$ \\
\hline 6 & $560 / 560$ & $109 / 107$ & $35 / 30$ & $3 / 2$ & $0 / 57$ & $3 / 7$ & $8 / 0$ & $6 / 4$ \\
\hline 7 & $400 / 400$ & $1 / 4 / 122$ & $20 / 34$ & $6 / 2$ & $40 / 74$ & $4 / 6$ & $8 / 5$ & $12 / 7$ \\
\hline 8 & $130 / 130$ & $104 / 108$ & $44 / 48$ & $2 / 2$ & $0 / 22$ & $5 / 6$ & $5 / 4$ & $4 / 3$ \\
\hline 9 & $400 / 230$ & $134 / 129$ & $31 / 37$ & $9 / 16$ & $11 / 40$ & $\mathrm{I} / 5$ & $12 / \mid$ & - \\
\hline 10 & $400 / 400$ & $102 / 123$ & $28 / 23$ & $\mathrm{I} / \mathrm{I}$ & $0 / 40$ & $6 / 7$ & $1 / 0$ & $5 / 2$ \\
\hline II & $560 / 560$ & $130 / 139$ & $40 / 50$ & $5 / 4$ & $0 / 74$ & $5 / 8$ & $5 / 0$ & $4 / 1$ \\
\hline 12 & $560 / 560$ & $124 / 134$ & $38 / 30$ & $1 / 2$ & $11 / 57$ & $\mathrm{I} / 2$ & $7 / 4$ & $2 / 1$ \\
\hline 13 & $560 / 560$ & $139 / 134$ & $26 / 28$ & $0 / 0$ & $0 / 40$ & $1 / 3$ & $7 / 3$ & $5 / 3$ \\
\hline
\end{tabular}

Abbreviations: JLOT, Judgment of Line Orientation Test.

${ }^{a}$ Contrast sensitivity: averaged scores in the Cambridge Low Contrast Gratings ${ }^{50}$; reading time: text reading time in seconds in a 180 -word reading test ${ }^{45}$; visual search: visual search on large visual displays, nonlateralized search time, and search errors ${ }^{51}$; line-orientation judgment: percentile rank in the JLOT $^{42}$; visuoconstructive disorder: number of correct items in the Mack-Levine test ${ }^{43}$; analog clock reading: number of correct items in the analog clock reading test; horizontal writing: median of the deviation from the objective horizontal in degrees.

processing time $(d f=2 ; F=13.51 ; P<.01)$ were found. There were significantly more correct items and reduced processing times in the posttraining session compared with the baseline sessions (all $P<.01$ ), whereas performance in the baseline sessions did not differ significantly (both $P>.40$ ).

For analog clock reading, a significant effect on the number of errors $(d f=2 ; F=31.36 ; P<.01)$ was found. Participants made significantly fewer errors in the posttraining session compared with the baseline sessions (both $P<.01)$, whereas performance in the baseline conditions did not differ significantly $(P>.35)$.

For horizontal writing, a significant effect on the medians of the deviation $(d f=2 ; F=24.12 ; P<.01)$ but not the intervals of uncertainty $(d f=2 ; F=2.25 ; P>.10)$ was revealed. Participants displayed smaller deviations in horizontal writing in the posttraining session compared with the baseline sessions (both $P<.01$ ), whereas performance in the baseline sessions did not differ significantly (both $P>.05$ ).

\section{Visual Tests Unrelated to Orientation Perception}

Measures of visual performance unrelated to orientation perception were equivalent before and after training (Table 2). Repeated-measures ANOVAs revealed no significant effect of training on contrast sensitivity $(d f=1 ; F=2.82 ; P$ $>.10)$, text reading performance $(d f=1 ; F=2.70 ; P>.10)$, or visual search performance (search time: $d f=1, F=.42$, $P>.50$; search errors: $d f=1, F=0.13, P>.90)$.

\section{Discussion}

We investigated effects of feedback-based perceptual training on orientation discrimination and further parameters of visuospatial performance in patients with profound visuospatial disorders. Clear improvements in orientation discrimination were obtained after only 4 weeks of therapy (11 sessions on average). There was no improvement across 2 baseline sessions, indicating that training effects related to spontaneous remission were unlikely. Improvements occurred not only in the trained orientation but transferred to all untrained orientations and the SVV/ SVH, suggesting a generalized benefit for spatial orientation perception. Training effects were stable in a follow-up session 8 weeks after training, showing that training led to persistent improvements. Furthermore, there was nearly perfect interocular transfer (measured in 2 participants) that is, equivalent improvements were found for both eyes, even when only 1 eye was trained. The substantial "far" transfer of improvement to further visuospatial and visuoconstructive tasks related to orientation perception (JLOT, ${ }^{43}$ Mack-Levine test ${ }^{44}$ horizontal writing, analog clock reading) along with the lack of improvement in visual tasks putatively unrelated to orientation discrimination (contrast sensitivity, visual search, and reading performance) supports the assumption of a generalized but still specific benefit of the training in tasks assumed to rely on orientation perception. 


\section{Putative Stages and Mechanisms of Improvements}

After training, participants showed reduced uncertainty intervals and constant errors for all orientations, indicating a progressive reduction of uncertainty along with a recalibration of perceptual tilts. Perceptual learning studies of orientation discrimination in unaffected controls suggest an involvement of early cortical visual areas but do not rule out a possible role of higher-level visual areas. ${ }^{54}$ Recent research using event-related potentials supports the role of several mechanisms of improvement in orientation discrimination at different levels of visuospatial processing. ${ }^{55,56}$ Several aspects of the present data provide information on the putative stages of visuospatial processing at which training affected performance. The nearly perfect interocular transfer observed in 2 participants strongly suggests a postchiasmatic locus for the improvement, beyond V1 where binocular interactions first occur in primate visual systems. ${ }^{57}$ This assumption is supported by the transfer of improvements to nontrained orientations and the SVV/SVH, indicating plasticity in higher regions of the dorsal or ventral stream relevant for orientation perception.

Interestingly, in unaffected participants, no or only limited transfer to nontrained orientations was reported. ${ }^{34,36}$ However, perceptual orientation learning might be dramatically different within injured brains. In intact brains operating at nearly perfect performance levels, training-related improvements are limited. In contrast, patients with focal brain damage might show a greater potential for modulations of visuospatial performance with practice. The recent decade has shown a considerable potential for improvements in, for example, spatial neglect. ${ }^{23}$

The considerable far transfer to further tasks related to orientation perception strengthens the assumption of a more central locus for the improvement at higher levels of visuospatial processing. It is important to note that only performance in visuospatial measures related to orientation discrimination improved with training. In contrast, performance in visual tasks unrelated to orientation discrimination-in which participants also showed substantial deficits-was not improved. These findings suggest that training effects are specific for orientation perception and related visuospatial functions but ineffective for unrelated visual functions and, thus, cannot be accounted for by unspecific factors such as increased motivation or alertness.

In unaffected controls, the (right) parietal cortex seems most critical for orientation processing. ${ }^{14-17}$ Nevertheless, several further brain regions, including V1, the lateral occipital cortex, superior temporal cortex, and subcortical structures also contribute to orientation discrimination. ${ }^{18,58,59}$ This distributed orientation processing network could be one potential explanation for the training benefits seen also in patients with parietal lesions. Recent research showed that mechanisms of plasticity after brain injury include the sprouting of fibers and formation of new synapses but also the redundancy of brain circuitry with parallel pathways performing similar functions. ${ }^{60,61}$ Possibly, repetitive feedbackbased training enhances the potential of dorsal or ventral stream areas relevant for orientation processing to take over functions of damaged parietal areas. Additionally, training might have stimulating effects on the damaged tissue and enhance mechanisms of neural repair. Research on functional reorganization supports the view that plasticity is not limited to primary sensory or motor areas - rather, the stroke-injured brain restores function via distributed neural networks involving brain regions upstream and downstream of the damaged region. ${ }^{62}$

The generalized improvements after repetitive feedbackbased training indicate that the damaged visual system is plastic at higher, possibly various, levels of visuospatial processing and shows considerable potential to reestablish functions with perceptual training.

\section{Clinical Relevance and Limitations of the Study}

We showed that persistent and generalized improvements in orientation discrimination can be achieved with a moderate training effort, which suggests that feedback-based perceptual training may be an effective and quickly accomplished strategy for aspects of visuospatial rehabilitation. It is important to note that training effects transferred to other visuospatial capacities essential for ADL. This generalization along with the persistence of improvements encourages the use of perceptual learning-related approaches in neurorehabilitation. It will be important to identify factors critical for the transfer to additional visual functions. In the present study, trialwise perceptual feedback might have been essential for training success and transfer, especially in participants with limited awareness regarding their deficits.

In this pilot study, we investigated a rather small sample of patients and did not assess performance compared with a control group. Furthermore, time since lesion onset, ranging from 12 to 28 weeks poststroke, does not rule out time-induced and spontaneous changes in performance, although repeated baseline assessments indicate that baseline performance was stable. Further research should investigate the potential of these training procedures with regard to generalization to improvements in performance parameters relevant for ADL in a well-powered, randomized controlled trial against another active treatment.

\section{Declaration of Conflicting Interests}

The author(s) declared no potential conflicts of interest with respect to the research, authorship, and/or publication of this article. 


\section{Funding}

The author(s) disclosed receipt of the following financial support for the research, authorship, and/or publication of this article: This work was supported by a Deutsche Forschungsgemeinschaft (DFG) grant to Georg Kerkhoff (IRTG 1457 “Adaptive minds”).

\section{References}

1. Kaplan J, Hier DB. Visuospatial deficits after right hemisphere stroke. Am J Occup Ther. 1982;36:314-321.

2. Dvorkin AY, Bogey RA, Harvey RL, Patton JL. Mapping the neglected space: gradients of detection revealed by virtual reality. Neurorehabil Neural Repair. 2012;26:120-131.

3. Trojano L, Fragassi NA, Chiacchio L, et al. Relationship between constructional and visuospatial abilities in normal subjects and in focal brain-damaged patients. J Clin Exp Neuropsychol. 2004;26:1103-1112.

4. Jesshope HJ, Clark MS, Smith DS. The Rivermead Perceptual Assessment Battery: its application to stroke patients and relationship with function. Clin Rehabil. 1991;5:115-122.

5. Hamsher K, Capruso DX, Benton A. Visuospatial judgement and right hemisphere disease. Cortex. 1992;28:493-495.

6. Kim Y, Morrow L, Passafiume D, Boller F. Visuoperceptual and visuomotor abilities and locus of lesion. Neuropsychologia. 1984;22:177-185.

7. Benton AL, Hannay HJ, Varney NR. Visual perception of line direction in patients with unilateral brain disease. Neurology. 1975;25:907-910.

8. De Renzi E, Faglioni P, Scotti G. Judgment of spatial orientation in patients with focal brain damage. J Neurol Neurosurg Psychiatry. 1971;34:489-495.

9. Pérennou D, Mazibrada G, Chauvineau V, et al. Lateropulsion, pushing and verticality perception in hemispheric stroke: a causal relationship? Brain. 2008;131:2401-2413.

10. Ferber S, Karnath HO. Parietal and occipital lobe contributions to perception of straight ahead orientation. J Neurol Neurosurg Psychiatry. 1999;67:572-578.

11. Tartaglione A, Cocito L, Bino G, Pizio N, Favale E. Further evidence for asymmetry of point localization in normals and unilateral brain damaged patients. Neuropsychologia. 1983;21:407-412.

12. Freedman M, Leach L, Kaplan E, Shulman KI, Delis DC. Clock Drawing: A Neuropsychological Analysis. New York, NY: Oxford University Press; 1994.

13. Young GC, Collins D, Hren M. Effect of pairing scanning training with block design training in the remediation of perceptual problems in left hemiplegics. J Clin Neuropsychol. 1983;5:201-212.

14. Tranel D, Vianna E, Manzel K, Damasio H, Grabowski T. Neuroanatomical correlates of the Benton Facial Recognition Test and Judgement of Line Orientation Test. J Clin Exp Neuropsychol. 2009;31:219-233.

15. Ng VW, Eslinger PJ, Williams SC, et al. Hemispheric preference in visuospatial processing: a complementary approach with fMRI and lesion studies. Hum Brain Mapp. 2000;10: 80-86.
16. Sack AT, Hubl D, Prvulovic D, et al. The experimental combination of rTMS and fMRI reveals the functional relevance of parietal cortex for visuospatial functions. Cogn Brain Res. 2001; 13:85-93.

17. Taira M, Kawashima R, Inoue K, Fukada H. A PET study of axis orientation discrimination. Neuroreport. 1998;9:283288.

18. Vandenberghe R, Dupont P, De Bruyn B, et al. The influence of stimulus location on the brain activation pattern in detection and orientation discrimination: a PET study of visual attention. Brain. 1996;119:1263-1276.

19. HierDB, MondlockJ, CaplanLR. Recovery ofbehavioralabnormalities after right hemisphere stroke. Neurology. 1983;33: 345-350.

20. Kerkhoff G. Rehabilitation of visuospatial cognition and visual exploration in neglect: a cross-over study. Restor $\mathrm{Neu}$ rol Neurosci. 1998;12:27-40.

21. Karnath HO, Rorden C. The anatomy of spatial neglect. Neuropsychologia. In press.

22. Jehkonen M, Laihosalo M, Kettunen J. Anosognosia after stroke: assessment, occurrence, subtypes and impact on functional outcome reviewed. Acta Neurol Scand. 2006;114:293-306.

23. Kerkhoff G, Schenk T. Rehabilitation of spatial neglect: an update. Neuropsychologia. In press.

24. Schindler I, Kerkhoff G, Karnath HO, Keller I, Goldenberg G. Neck muscle vibration induces lasting recovery in spatial neglect. J Neurol Neurosurg Psychiatry. 2002;73:412-419.

25. Mizuno K, Tsuji T, Takebayashi T, et al. Prism adaptation therapy enhances rehabilitation of stroke patients with spatial neglect: a randomized, controlled trial. Neurorehabil Neural Repair. 2011;25:711-720.

26. Saevarsson S, Kristjánsson Á, Hildebrandt H, Halsband U. Prism adaptation improves visual search in hemispatial neglect. Neuropsychologia. 2009;47:717-725.

27. Karnath HO. Subjective body orientation in neglect and the interactive contribution of neck muscle proprioceptive and vestibular stimulation. Brain. 1994;117:1001-1012.

28. Mattingley JB, Bradshaw JL, Bradshaw JA. Horizontal visual motion modulates focal attention in left unilateral spatial neglect. J Neurol Neurosurg Psychiatry. 1994;57:1228-1235.

29. Keller I, Lefin-Rank G, Lösch J, Kerkhoff G. Combination of pursuit eye movement training with prism adaptation and arm movements in neglect therapy: a pilot study. Neurorehabil Neural Repair. 2009;23:58-66.

30. Nyfeller T, Cazzoli D, Hess CW, Müri RM. One session of repeated parietal theta burst stimulation trains induces longlasting improvement of visual neglect. Stroke, 2009;40:27912796.

31. Weinberg J, Piasetsky E, Diller L, Gordon W. Treating perceptual organization deficits in nonneglecting RBD stroke patients. J Clin Exp Neuropsychol. 1982;4:59-74.

32. Goldstone RL. Perceptual learning. Annu Rev Psychol. 1998;49:585-612.

33. Fine I, Jacobs RA. Comparing perceptual learning across tasks: a review. J Vis. 2002;2:190-203. 
34. Schoups AA, Vogels R, Orban GA. Human perceptual learning in identifying the oblique orientation: retinotopy, orientation specificity and monocularity. J Physiol. 1995;483:797-810.

35. Shiu LP, Pashler H. Improvement in line orientation discrimination is retinally local but dependent on cognitive set. Percept Psychophys. 1992;52:582-588.

36. Vogels R, Orban GA. The effect of practice on the oblique effect in line orientation judgements. Vision Res. 1985;25:1679-1687.

37. Fahle M. Perceptual learning: specificity versus generalization. Curr Opin Neurobiol. 2005;15:154-160.

38. Fahle M, Skrandies W. An electrophysiological correlate of learning in motion perception. Ger J Ophthalmol. 1994;3:427-432.

39. Sagi D, Tanne D. Perceptual learning: learning to see. Curr Opin Neurobiol. 1994;4:195-199.

40. Polat U. Making perceptual learning practical to improve visual functions. Vision Res. 2009;49:2566-2573.

41. Leonards U, Rettenbach R, Nase G, Sireteanu R. Perceptual learning of highly demanding visual search tasks. Vision Res. 2002;42:2193-2204.

42. Ball K, Sekuler R. Direction-specific improvement in motion discrimination. Vision Res. 1987;27:953-965.

43. Benton AL, Hamsher KD, Varney NR, Spreen O. Judgement of Line Orientation. New York, NY: Oxford University Press; 1983.

44. Mack JL, Levine RN. The basis of visual constructional disability in patients with unilateral cerebral lesions. Cortex. 1981;17:515-532.

45. Kuhn C, Heywood CA, Kerkhoff G. Oblique spatial shifts of subjective visual straight ahead orientation in quadrantic visual field defects. Neuropsychologia. 2010;48:3205-3210.

46. Kerkhoff G, Münßinger U, Eberle-Strauss G, Stögerer E. Rehabilitation of hemianopic alexia in patients with postgeniculate visual field disorders. Neuropsychol Rehabil. 1992;2: 21-42.

47. Huber W, Poeck K, Weniger D, Willmes K. Der Aachener Aphasie Test (AAT). Göttingen, Germany: Hogrefe; 1983.

48. Kerkhoff G, Marquardt C. VS: a new computer program for detailed offline analysis of visual-spatial perception. J Neurosci Methods. 1995;63:75-84.

49. Engen T. Psychophysics I: discrimination and detection. In: Kling JW, Riggs L, eds. Woodworth and Schlossberg's
Experimental Psychology. London, UK: Methuen; 1971: 11-86.

50. Kerkhoff G, Marquardt C. Standardised analysis of visualspatial perception after brain damage. Neuropsychol Rehabil. 1998;8:171-189.

51. Benton AL, Sivan AB, Hamsher K, Varney NR, Spreen O. Contributions to Neuropsychological Assessment: A Clinical Manual. New York, NY: Oxford University Press; 1983.

52. Wilkins AJ, Della Sala S, Somazzi L, Nimmo-Smith I. Agerelated norms for the Cambridge Low Contrast Gratings, including details concerning their design and use. Clin Vision Sci. 1988;2:201-212.

53. Kerkhoff G, Münßinger U, Meier EK. Neurovisual rehabilitation in cerebral blindness. Arch Neurol. 1994;51:474-481.

54. Seitz A, Watanabe T. A unified model for perceptual learning. Trends Cogn Sci. 2005;9:329-334.

55. Ding Y, Song Y, Fan S, Qu Z, Chen L. Specificity and generalization of visual perceptual learning in humans: an event-related potential study. Neuroreport. 2003;14: 587-590.

56. Song Y, Peng D, Lu C, et al. An event-related potential study on perceptual learning in grating orientation discrimination. Neuroreport. 2007;18:945-948.

57. Hubel DH, Wiesel TN. Receptive fields and functional architecture of monkey striate cortex. J Physiol. 1968;195: 215-243.

58. Faillenot I, Sunaert S, Van Hecke P, Orban GA. Orientation discrimination of objects and gratings compared: an fMRI study. Eur J Neurosci. 2001;13:585-596.

59. Shikata E, Hamzei F, Glauche V, et al. Functional properties and interactions of the anterior and posterior intraparietal areas in humans. Eur J Neurosci. 2003;17:1105-1110.

60. Duffau H. Brain plasticity: from pathophysiological mechanisms to therapeutic applications. J Clin Neurosci. 2006;13: 885-897.

61. Nudo RJ. Neural bases of recovery after brain injury. J Commun Disord. 2011;44:515-520.

62. Murphy TH, Corbett D. Plasticity during stroke recovery: from synapse to behavior. Nat Rev Neurosci. 2009;10: 861-872. 